Article

\title{
Modeling GATAD1-Associated Dilated Cardiomyopathy in Adult Zebrafish
}

\author{
Jingchun Yang ${ }^{1}$, Sahrish Shah ${ }^{1}$, Timothy M. Olson ${ }^{2,3}$ and Xiaolei Xu ${ }^{1,2, *}$ \\ 1 Department of Biochemistry and Molecular Biology, Mayo Clinic College of Medicine, \\ 200 First St. SW Rochester, MN 55905, USA; yang.jingchun@mayo.edu (J.Y.); shah.sahrish@mayo.edu (S.S.) \\ 2 Department of Internal Medicine, Division of Cardiovascular Diseases, Mayo Clinic College of Medicine, \\ 200 First St. SW Rochester, MN 55905, USA; Olson.Timothy@mayo.edu \\ 3 Department of Pediatrics and Adolescent Medicine, Division of Pediatric Cardiology, \\ Mayo Clinic College of Medicine, 200 First St. SW Rochester, MN 55905, USA \\ * Correspondence: xu.xiaolei@mayo.edu; Tel.: +1-507-284-0685; Fax: +1-507-538-6418
}

Academic Editors: Georg Vogler and Rolf Bodmer

Received: 1 December 2015; Accepted: 22 January 2016; Published: 26 January 2016

\begin{abstract}
Animal models have played a critical role in validating human dilated cardiomyopathy (DCM) genes, particularly those that implicate novel mechanisms for heart failure. However, the disease phenotype may be delayed due to age-dependent penetrance. For this reason, we generated an adult zebrafish model, which is a simpler vertebrate model with higher throughput than rodents. Specifically, we studied the zebrafish homologue of GATAD1, a recently identified gene for adult-onset autosomal recessive DCM. We showed cardiac expression of gatad1 transcripts, by whole mount in situ hybridization in zebrafish embryos, and demonstrated nuclear and sarcomeric I-band subcellular localization of Gatad1 protein in cardiomyocytes, by injecting a Tol2 plasmid encoding fluorescently-tagged Gatad1. We next generated gatad1 knock-out fish lines by TALEN technology and a transgenic fish line that expresses the human DCM GATAD1-S102P mutation in cardiomyocytes. Under stress conditions, longitudinal studies uncovered heart failure (HF)-like phenotypes in stable $\mathrm{KO}$ mutants and a tendency toward HF phenotypes in transgenic lines. Based on these efforts of studying a gene-based inherited cardiomyopathy model, we discuss the strengths and bottlenecks of adult zebrafish as a new vertebrate model for assessing candidate cardiomyopathy genes.
\end{abstract}

Keywords: adult zebrafish; cardiomyopathy; gatad1; TALEN; transgenic fish

\section{Introduction}

Cardiomyopathy refers to cardiac disease that is associated with structural changes of the myocardium upon extrinsic stresses such as ischemia and hypertension, and intrinsic stresses such as genetic mutations, many of which ultimately lead to heart failure [1]. Depending on the nature of the biomechanical stressors, various remodeling phenotypes can occur, including hypertrophic cardiomyopathy (HCM), dilated cardiomyopathy (DCM), and restrictive cardiomyopathy (RCM). Human genetic studies have identified mutated genes responsible for $50 \%-70 \%$ of $\mathrm{HCM}, 30 \%-50 \%$ of DCM, and a small fraction of RCM [2,3], which create opportunities to pinpoint disrupted signaling pathways and seek therapeutic strategies. Beyond hypothesis-based candidate gene approaches, the development of genomic technologies including genome-wide association studies, family-based locus mapping, whole-exome sequencing, and chromosomal microarray have facilitated discovery of novel, unsuspected susceptibility genes [4]. However, these strategies may yield multiple plausible candidate genes in individual families and/or require a higher burden of proof to establish causality. An efficient animal model with high throughput is needed to assess these candidate genes and to expedite the gene discovery process. 
The mouse is currently the preferred animal to model cardiomyopathy, but the associated high costs and low throughput limit its application for discovering and validating new cardiomyopathy genes. As reviewed by other papers in this special issue, Drosophila is a highly efficient invertebrate model that has contributed significantly to our understanding of cardiogenesis and adult heart diseases. However, the tubular structure of a fly heart and the fact that $39 \%$ of human genes are missing in fly genome impose significant limitations on assessing candidate genes for cardiomyopathy $[5,6]$. In contrast, the zebrafish heart can be thought of as a simplified human heart, consisting of a single atrium, ventricle, and outflow tract [7]. Major cardiac structures including the myocardium, endocardium, epicardium and cardiac valves are conserved in this vertebrate model. Besides structural conservation, conservation at the molecular level has been supported by a comprehensive scan of the zebrafish genome to identify homologues of known human DCM genes. Ninety-six percent of known human DCM genes (49 of 51) have a corresponding zebrafish homologue, of which 30 have a single homologue [8]. For 14 out of the remaining 19 genes with multiple homologues, a single zebrafish homologue has been prioritized from other homologues by scoring its cardiac abundance and enrichment based on RNAseq analysis [8]. Together, these data lay a foundation for generating genetic cardiomyopathy mutants in adult zebrafish.

The use of zebrafish as an animal model to study cardiomyopathy was initially reported in 2002, when Xu et al. identified a titin mutant and Sehnert et al. cloned a tnnt2 mutant, representing two embryonic zebrafish forms of cardiomyopathy $[9,10]$. Because of efficient technologies that can be applied during zebrafish embryogenesis, such as morpholinos to knock-down gene expression and chemical screening platforms to search for phenotypic modification, zebrafish have been successfully used to validate candidate genes identified by human genetic studies [11,12], discover new cardiomyopathy genes [13], and seek potential therapeutic strategies [14,15]. However, fish embryos have intrinsic limitations. First, the short developmental time window prevents fish embryos from faithfully recapitulating the pathogenic process of cardiomyopathy, which typically involves age-dependent penetrance and gradual progression to overt heart failure in adulthood. Second, the inheritance patterns of cardiomyopathy can be diverse, including autosomal dominant, autosomal recessive (homozygous and compound heterogeneous), and X-linked recessive, and difficult to recapitulate by assessing embryonic phenotypes upon near-null gene depletion. It is estimated that only $5 \%-10 \%$ of genes exhibit embryonic-lethal phenotypes upon depletion [16]. Third, there are significant concerns about the specificity of the morpholino technology, which might incur pathological changes different from stable mutants $[17,18]$. Having recognized the shortcomings of the fish embryo, Sun et al. started to explore whether cardiac remodeling exists in an adult zebrafish heart [19]. Detailed characterization of $\operatorname{tr} 265$, a mutant with a defective band 3 gene [20], showed that high-output cardiac stress from chronic anemia causes significant enlargement of the ventricular chamber. Hallmarks of cardiomyopathy, including reduced ejection fraction, muscular disarray, and fetal gene reactivation, were evident $[19,21]$. At the cellular level, cardiomyocyte (CM) hypertrophy and activated CM proliferation have been detected. Ding et al. reported that injection of doxorubicin (DOX), a widely used anticancer drug that induces cardiomyopathy in humans and rodent models [22,23], likewise induces ventricular enlargement in adult zebrafish and is associated with the hallmarks of cardiomyopathy [24]. Strong activation of $\mathrm{CM}$ apoptosis and unchanged $\mathrm{CM}$ proliferation was noted, indicating that the pathogenic mechanisms differed between the DOX and anemia models. The successful generation of two acquired-cardiomyopathy models with different pathogenesis demonstrated that the simple zebrafish heart is likely complex enough to model different types of cardiomyopathy. Consistent with this concept, a transgenic fish line that expresses a human 2057del2 mutation in the gene encoding Plakoglobin recapitulates perspectives of arrythmogenic cardiomyopathy [25]. These reports justify the present work to further explore genome editing technology to model cardiomyopathy in adult zebrafish.

Historically, most zebrafish stable mutants have been generated by either large scale mutagenesis screening or TILLING technology [26-28]. Both strategies depend on generating random mutations in the whole genome, which is only feasible by collaborative efforts in large research centers. 
The emergence of genome editing technology, as represented by transcription activator-like effector nuclease (TALEN) and/or CRISPR/Cas9-based technologies [29-31], have made it possible to genetically edit specific genes in an individual laboratory. In addition to nonsense truncation mutations, missense mutations can also be efficiently generated by using TALEN-based knock-in (KI) technology and/or transgenic technology in zebrafish [32-34].

GATA zinc finger domain containing 1 (Gatad1), also called (ocular development-associated gene (ODAG), was first cloned from mouse, in which Gatad1 is ubiquitously expressed in all tissues with the highest levels in testis and lung. During development, mouse Gatad1 has a dynamic expression in the eye and overexpression of Gatad1 impairs retinal development $[35,36]$. Through homozygosity mapping and exome sequencing, Theis et al. identified a GATAD1 mutation from patient cohorts with autosomal recessive dilated cardiomyopathy [37]. Interestingly, GATAD1 is highly overexpressed in female idiopathic dilated cardiomyopathy (IDCM) patients comparing to male patients, suggesting GATAD1 might contribute to gender difference in DCM patients [38].

Given that genetic models of cardiomyopathy in adult zebrafish have scarcely been reported, we leveraged genome editing technology to systematically generate stable mutants for known cardiomyopathy genes. Based on our experience with the Golden Gate custom array platform [39], TALEN constructs for five to 10 genes can be generated in two weeks, and stable knock-out (KO) zebrafish mutants can be obtained for most of them in about three months (data not published) [40]. Here, we report our study of GATAD1, a recently established gene for human DCM. In a family with adult-onset DCM, a homozygous recessive missense mutation-S102P-was discovered by locus mapping and whole exome sequencing [37]. We determined the tissue distribution of the gatad1 transcript and the subcellular localization of the zebrafish Gatad1 protein. We then generated stable gatad1 knock-out fish by TALEN technology and transgenic fish lines that overexpressed human GATAD1-S102P. Heart failure-like phenotypes were noted in these genetically manipulated fish after stress. Based on these data, we review adult zebrafish as an emerging vertebrate model for assessing candidate genes for cardiomyopathy and discuss the bottlenecks of this model that need to be overcome.

\section{Experimental Section}

\subsection{Zebrafish Lines and Maintenance}

Wild type WIK fish were used for this study. The IACUC approval number is A32413.

\subsection{Bioinformatics Analysis}

DSgene was used to obtain the alignments and phylogenetic tree for gatad1 sequences from different species. The Gatad1 protein sequences from the following species have been used for alignment: Homo sapiens (NM_021167), Rattus norvegicus (XP_575359); Mus musculus (NP_080309), Xenopus laevis (NM_001087134), Danio rerio (NM_001040364), Drosophila melanogaster (NP_569851), Oncorhynchus mykiss (CDQ77377), Oreochromis niloticus (XP_003444124), Takifugu rubripes (XP_003969420), Oryzias latipes (XP_004074272), Astyanax mexicanus (XP_007245785), Pundamilia nyererei (XP_005728478), Xiphophorus maculatus (XP_005797418), Haplochromis burtoni (XP_005917525), Neolamprologus brichardi (XP_006787902).

\subsection{Whole-Mount in Situ Hybridization}

Whole mount in situ hybridization was performed as previously described [41]. The following primer pair was used for generating gatad1 riboprobes: forward primer $5^{\prime}$-TATCATGGTAAAAC ATACGGGACC- $3^{\prime}$ and reverse primer $5^{\prime}$-GGTAATACGACTCACTATAGGCATGGACTCTGATGT GATGATCGTTG-3'.

\subsection{Immunostaining}

myl7: Gatad1-GFP construct was injected into 1-cell staged fish embryo. GFP signal starts to express at $2 \mathrm{dpf}$, when the heart was dissected from fish embryo and stained as described before [42]. 
The primary antibodies are $\alpha$-actinin (Sigma, 1:200, St. Louis, MO, USA), egfp (Invitrogen, 1:200, Grand Island, NY, USA), myomesin (DSHB, 1:20, Iowa City, IA, USA), and then Alexa-conjugated secondary antibodies were applied (Invitrogen, 1:1000). Alexa conjugated phalloidin (Invitrogen, 1:200) was used to stain actin filament.

Frozen sections from adult fish hearts were stained with Alexa conjugated phalloidin (Invitrogen, 1:200) as described before [19].

\subsection{Real-Time Reverse Transcription PCR}

Different tissues including brain, heart, intestine, liver, muscle and spleen were dissected from adult zebrafish, and homogenized in Trizol using the Advanced Blender system with RNase-free $0.5 \mathrm{~mm}$ steel beads (Advanced Blending Solution, Wallace, MI, USA). Total RNAs were extracted from these tissues using the RNeasy mini kit (Qiagen, Frederick, MD, USA), and RNAs were used as templates for reverse transcription using the SuperScript ${ }^{\circledR}$ III Reverse Transcription kit (Invitrogen, Grand Island, NY, USA). Real-time PCR was performed with the LightCycler ${ }^{\circledR} 480$ System (Roche, Indianapolis, IN, USA) using the following primers. For gatad1, forward primer: 5'-CAGGA AATACACAGACGCTC-3' and reverse primer: 5'-AGTACTGATCCTGGACGAATC-3'; For 18s, forward primer: 5'-TCGCTAGTTGGCATCGTTTATG-3' and reverse primer: 5'-CGGAGGTTCG AAGACGATCA-3'; for gapdh, forward primer: 5'-CCACCCATGGAAAGTACAAG-3' and reverse primer: $5^{\prime}$-CTCTCTTTGCACCACCCTTA-3'; for $n p p b$, vmhc and $v m h c l$, the primer sequences have been described before [8].

\subsection{Generation of TALEN Mutants}

To generate TALEN knock-out zebrafish, the 2nd exon of gatad1 (ENSDARG00000027612) was selected as the targeted site. This exon corresponds to the human exon that has the S102P mutation. The TALEN RNA was generated using a Golden Gate kit (Addgene, Cambridge, MA, USA) according to the instructions from the manufacturer. Briefly, the targeted exon was amplified and sequenced to ensure that no genetic variants were present in the targeted region. The TALEN RNA binding site was predicted using the Zifit website [43]. Based on the binding sequences, final constructs that encoded the desired TALEN RNAs were generated through recombining several plasmids provided in the Golden Gate kit. Using the linearized final construct as a template, the TALEN RNA was synthesized using an mMESSAGE mMachine T3 kit (Ambion, Pittsburgh, PA, USA). For the allele with the 4 nucleotides deletion, the binding sequence for the TALEN left arm is $5^{\prime}$-TGCAGTCCAAACAGGAAA- ${ }^{\prime}$, and the binding sequence for the right arm is 5'-AGGTTAAGAAGCACTAA-3'. The targeted site contains a recognition site for the $\mathrm{Hgal}$ restriction enzyme. For the allele with a 13 nucleotide deletion, the binding sequence for the TALEN left arm is $5^{\prime}$-TTAAGAAGCACTAAGTAT-3', for the right arm is 5'-AAAAAAAAGTCTCCACCA-3', and the central targeted site contains a cut site for MspI. The primers for genotyping of both alleles are: forward: 5'-TTCTTTGCAGTCCAAACAGG-3' and reverse: 5'-AAAGAGGGTCCAACCAGGTACTAG-3'.

To generate stable TALEN mutant fish, TALEN RNAs were injected at $10 \mathrm{ng} / \mu \mathrm{L}$ into single-cell staged embryos. Founder fish containing deletions were identified by genotyping with restriction enzymes, followed by sequencing to determine the precise molecular nature of the genomic change. Founder fish with the desired mutation were outcrossed to produce an F1 generation, which was then used for breeding to generate homozygous mutants. Homozygous mutant fish were identified by restriction fragment length polymorphism based genotyping.

\subsection{Generation of Transgenic Fish Lines}

To generate the Tol2 (myl7:gatad1-gfp) construct, zebrafish gatad1 open reading frame sequence (NM_001040364) was cloned into a gateway middle entry clone pENTR1A (Invitrogen) before it was recombined with the Tol2 destination vector, p5E-myl7 vector, and the p3E-gfp vector [34]. To generate Tol2 (myl7:GATAD1-HA IRES-GFP) and Tol2 (myl7:GATAD1-HA-S102P IRES-GFP) 
constructs, human GATAD1 (NM_021167) was cloned into the pENTR1A vector using the following primer pair: forward: 5'-TTAGGATCCGCCACCATGCCGCTGGGCCTGAAGCCCACC-3' and reverse: 5'-GGTGAATTCTCAAGCGTAATCCGGAACATCGTATGGGTACATCAAATGGTTGG CAACTGATTCC- $3^{\prime}$. An HA tag was included at the carboxyl terminal of GATAD1. Next, the S102P mutation was introduced into the wild type GATAD1 by two-step PCR using the following primers:

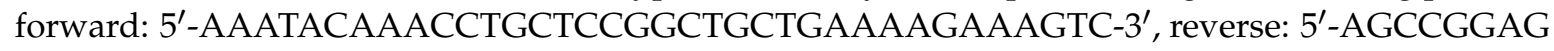
CAGGTTTGTATTTAGTGTTTCTGAGCCG-3', and the primer pairs used for cloning wild type GATAD1. Finally, the pENTR1A-based constructs containing either wild type GATAD1 or mutant GATAD1 were recombined into the Tol2 destination expression vector, together with the p5E-myl7 and p3E-IERS-GFP vectors using the Gateway system (Invitrogen). To generate stable transgenic fish, the Tol2 expression constructs together with transposase RNA were injected into fish embryos at the 1-cell stage. The injected fish were raised to the adult stage and the positive fish were selected based on the GFP reporter.

\subsection{Stressing Fish with a High-Cholesterol Diet}

A 4\% cholesterol diet was made as previously descripted [44,45]. Briefly, $4 \mathrm{~g}$ cholesterol (Sigma, St. Louis, MO, USA) was dissolved in $200 \mathrm{~mL}$ ether before being mixed with $100 \mathrm{~g}$ of artificial artemia. The mixture was put close to the ventilation opening in the hood to evaporate until completely dry. The fish were fed with this high cholesterol diet twice a day beginning at 5 weeks of age.

\subsection{Stressing Zebrafish Embryos with Ethanol}

To impose a cardiomyopathy-inducing stressor in zebrafish, we treated the fish with ethanol during embryogenesis [46]. Briefly, the fish embryos were immersed in $0.3 \%$ ethanol from $2 \mathrm{hpf}$ to $48 \mathrm{hpf}$. The embryos that did not show a phenotype after ethanol treatment were raised up to the adult stage in normal fish water.

\subsection{Swimming Capacity Test}

The fish were starved for $24 \mathrm{~h}$ before being placed in a swim tunnel respirometer (Mini Swim-170, Loligo Systems, Tjele, Denmark). After being acclimated to the respirometer at a speed $9 \mathrm{~cm} / \mathrm{s}$ (200 rpm) for $20 \mathrm{~min}$, the fish were challenged by swimming against the stream with increased water speed with an increment of $8.66 \mathrm{~cm} / \mathrm{s}$ (100 rpm) (Ui), with each speed lasting for $2.5 \mathrm{~min}$ (Tii). When a fish became exhausted and unable to resume swimming from the downstream screen of the swim chamber, the maximum speed (Uii) and the passing time (Ti) within the cycle was recorded for this particular fish. After all fish were exhausted, the fish were allowed to recover for about $30 \mathrm{~min}$ and body length measurements (BL) were then determined. The critical swimming speed (Ucri) was calculated according to the following equation [47,48].

$$
\mathrm{Ucrit}=\mathrm{Ui}+[\mathrm{Uii}(\mathrm{Ti} / \mathrm{Tii})]
$$

\subsection{Assessment of Fish Survival Curve And Ventricle Area}

Fish deaths were recorded weekly starting from 3 months of age. The survival curve was drawn and the $p$ value was calculated using JMP software. Ventricle area was measured in age matched and control and mutant fish group as described before [19].

\subsection{Statistical Analysis}

The values displayed in each graph are mean \pm standard deviation. The non-parametric Wilson test was used to compare the mean from two different groups using JMP software (version 11, Cary, NC, USA). $p<0.05$ was regarded as a significant difference between the two groups. 


\section{Results}

\subsection{Comparison of Gatad1 Gene Sequences between Zebrafish and Humans}

In humans, GATAD1 is located on chromosome 7q21-7q22 and encodes a 269 amino acid protein. The corresponding zebrafish orthologue gatad1 is located on chromosome 19 and encodes a 242 amino acid protein. There is a single transcript listed in the Ensembl database for zebrafish gatad1 consisting of five exons (ENSDARG00000027612), which is similar to human GATAD1 (ENSG00000157259). The genomic DNA sequence in the last four exons has $70 \%-82 \%$ sequence identity between zebrafish and human. The genomic DNA in exon 1 of zebrafish gatad1 has 400 nucleotides, 360 of which have no significant similarity and 41 of which have $80 \%$ identity to corresponding sequence in exon 1 from the human GATAD1 gene. The translation start site is localized to the 1st exon, while the peptide surrounding the human S102P mutation is encoded by the 2nd exon. At the protein level, there is $74 \%$ sequence identity between human GATAD1 and zebrafish Gatad1 proteins (Figure 1A). A gatad1 orthologue can also be identified in the invertebrate Drosophila, but the sequence of the encoded protein is much more divergent from human GATAD1 (Figure 1B). Although there is remarkable conservation in the C-terminal half, the N-terminal half of the Gatad1 peptide including the domain surrounding the human S102P mutation is not conserved, rendering concern whether pathological changes resulting from the S102P mutation can be recapitulated in Drosophila. No GATAD1 homologue was found in lower model organisms such as Caenorhabditis elegans and Saccharomyces cerevisiae.

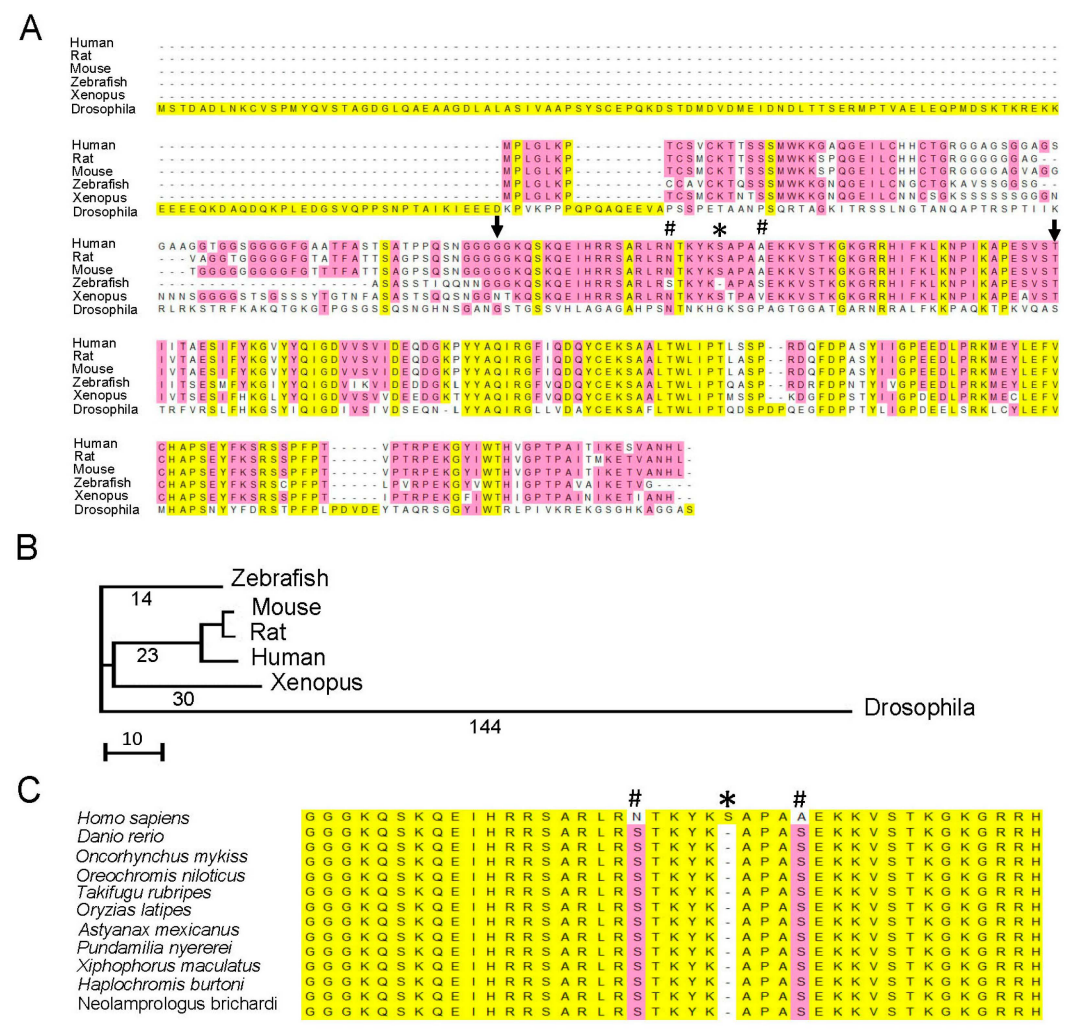

Figure 1. Gatad1 is highly conserved between human and zebrafish. (A) Alignment of human GATAD1 protein sequence and five animal species. The human S102P mutation is localized to a region highly conserved in most animal models except Drosophila. Zebrafish does not have a serine residue in the position corresponding to human S102, but it has serine residues in positions corresponding to human N 97 and A106. \# Human N 97 and A106; * Human S 102; arrows, range of highly conserved sequence from human G79 to I136; (B) The phylogenetic tree generated by comparing Gatad1 protein sequences in human and 5 animal species; (C) The alignment of peptide sequences flanking human S102 between human and fish species. All fish species have serine residue in the positions corresponding to human N 97 and A106, but not S102. 
In mammals, the peptide sequence surrounding the human GATAD1-S102P mutation, ranging from G79 to I136, is 100\% conserved (Figure 1A). Interestingly, zebrafish Gatad1 is also highly conserved in this domain except for the residue corresponding to S102 in human GATAD1. In fact, zebrafish Gatad1 does not contain the serine residue in this position, but has an additional two serine residues in positions that correspond to N97 and A106 in human GATAD1. This unique sequence pattern is found in Gatad1 proteins from all fish species (Figure 1C), indicating an early evolutionary event that occurred when mammals started to diverge from fish.

\subsection{Tissue Distribution of the Gatad1 mRNA}

We assessed gatad1 mRNA expression in adult zebrafish tissues via real time RT-PCR. The relative expression of gatad1 in heart is lower than in brain but higher than in intestine, liver, muscle and spleen (Figure 2A). We also examined absolute gatad1 mRNA expression using our RNAseq data generated from both embryonic and adult zebrafish hearts [8]. We found that the expression of gatad1 is 2.5 reads per kilobase per million reads (RPKM) in an embryonic fish heart and 1.1 RPKM in an adult fish heart. While our data indicated that although gatad1 does not have heart-enriched expression, it can be included with the 10,000 cardiac genes with RPKM $>0.3$, i.e., it has a meaningful absolute expression level in the heart (Figure 2B).

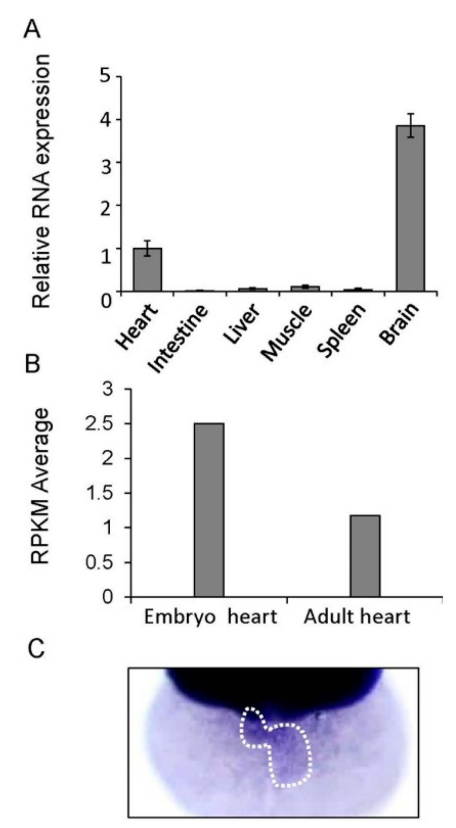

Figure 2. Assessment of the cardiac expression of gatad1 in zebrafish. (A) The relative expression level of gatad1 in different tissues were revealed by real-time RT-PCR using $18 \mathrm{~s}$ as internal control. Cardiac expression was defined as 1 and used as a reference. The brain has the highest gatad1 expression; (B) Different expression levels of gatad1 in embryonic and adult zebrafish hearts were revealed by RNA sequencing. gatad1 expression in the embryonic heart is higher than that in the adult heart; (C) Cardiac expression of gatad1 transcripts was also revealed by in situ hybridization in $2 \mathrm{dpf}$ zebrafish embryos. RPKM: number of reads per kilobase per million reads.

Whole-mount in situ hybridization (ISH) is a convenient technology in zebrafish embryos that can reveal tissue-specific expression patterns for a gene of interest. Cardiac expression of the gatad1 transcript can be detected at 2 days post fertilization (dpf) after overstaining the embryos (Figure 2C). In addition to cardiac expression, we detected strong gatad1 expression in the head, and weak expression in notochord, gut and pectoral fin (Supplementary Figure S1). 


\subsection{Subcellular Expression Pattern of Gatad1 Protein in the Nucleus and Sarcomeric I Band}

To determine the subcellular expression of the Gatad1 protein in the zebrafish heart, we generated a construct that encodes a GFP-tagged protein. After injecting the plasmid into a single cell stage zebrafish embryo, we isolated zebrafish hearts at $48 \mathrm{~h}$ post injection and determined the localization of GFP signal that is mosaically expressed. We noted strong GFP signals in the nuclei as well as the myofibril network, as revealed by co-staining Actin filaments with phalloidin (Figure 3A). Besides a diffuse expression pattern within cardiomyocytes, the Gatad1-GFP signal exhibited a striated pattern within the myofibril network. Double immunostaining studies demonstrated that the striated expression of Gatad1-GFP is attributable to co-localization with the I-band, as revealed by phalloidin, partial co-localization with the Z-disc, as revealed by an $\alpha$-actinin antibody, but not co-localization with the M-line, as revealed by a myomesin antibody (Figure 3B,C). Together, these data indicate that Gatad1-GFP is localized to both the nucleus and the I-band region of myofibrils.

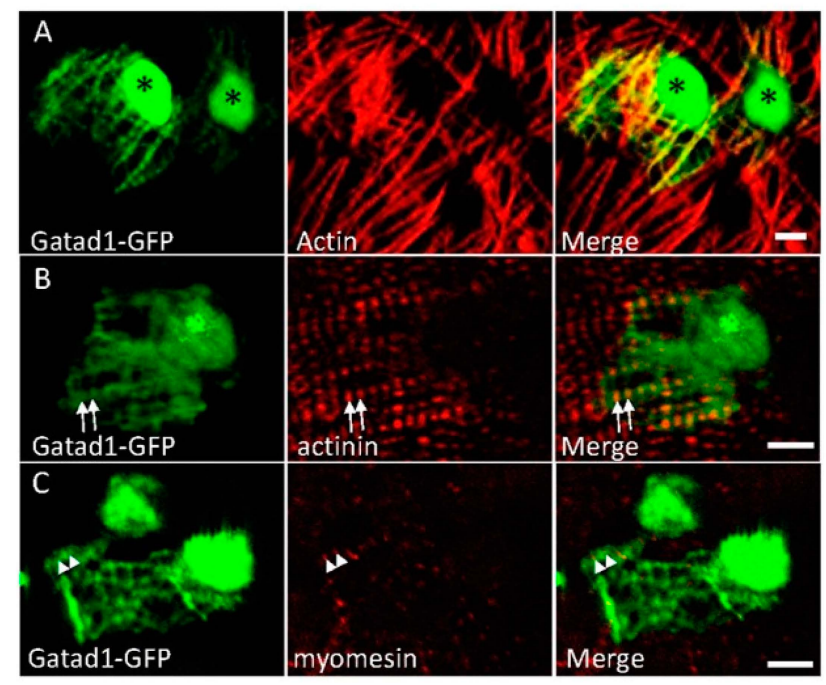

Figure 3. Subcellular localization of the Gatad1 protein in the embryonic zebrafish heart. After the myl7:gatad1-GFP construct was injected into 1-cell staged embryos, hearts from $2 \mathrm{dpf}$ embryos were dissected for immunostaining and imaging. (A) Gatad1-GFP shows strong expression in nuclei and relatively weak expression in myofibrils, overlapping with Actin as revealed by phalloidin staining; (B) Gatad1-GFP partially overlaps with Z-discs marked by Actinin (arrows); (C) Gatad1-GFP forms alternatively striated patterns with M-line marked by Myomesin (arrowheads). * Nucleus; Scale bar $5 \mu \mathrm{m}$.

\subsection{Heart Failure-Like Phenotypes in Gatad1 Knock-out Fish}

To further validate GATAD1 as a cardiomyopathy gene, we generated gatad1 homozygous mutants by designing a pair of TALEN RNAs targeting the 2nd exon of the zebrafish gatad1 gene (Figure 4A). Two mutant alleles, gatad $1^{4 n t d e l}$ and gatad1 $1^{13 n t d e l}$, were identified, which contained 4- and 13-base pair deletions that created different frameshifts. These small deletions would result in premature stop codons during translation, resulting in truncated Gatad1 proteins (Figure 4A). In fact, gatad1 RNA was reduced by $98 \%$ in fish heart isolated from homozygous gatad1 ${ }^{13 n t d e l}$, presumably due to nonsense-mediated mRNA decay (Figure 4B). Both gatad1 knock-out mutant alleles survived to adult without any visually apparent phenotypes. The gatad $1^{13 n t d e l}$ mutant was used for further experimentation. To determine if these fish were vulnerable to heart failure, we imposed stress by feeding a $4 \%$ cholesterol diet starting from 2 months of age. On this high cholesterol diet, gatad1 homozygous mutant fish started to show reduced swimming capacity at 1.5 years of age (data not shown). To impose additional stress, gatad1 mutant fish and age matched controls were treated with 
$0.3 \%$ ethanol during their embryonic stage from $2 \mathrm{hpf}$ to $48 \mathrm{hpf}$, and then fed with $4 \%$ cholesterol starting from 2 months of age. Besides the reduced swimming capacity, we observed that gatad 1 homozygous mutant fish started to die after approximately 7 months of age and exhibited a significant reduction in survival rate comparing to age-matched wild type control fish (Figure 4C,D). Real-time RT-PCR revealed significant induction of $n p p b$ and vmhc in gatad1 KO fish heart comparing to wild type fish heart. Nppb and vmhc are two fetal genes that have been used as molecular markers for hypertrophy [8], supporting a heart failure (HF) phenotypes (Figure 4B). Quantification of the heart size indicated a tendency for the group stressed with both ethanol and high cholesterol to show enlarged hearts $(p=0.2)$ (Figure 4E), but the group stressed with only a high cholesterol diet did not show enlarged hearts (data not shown).

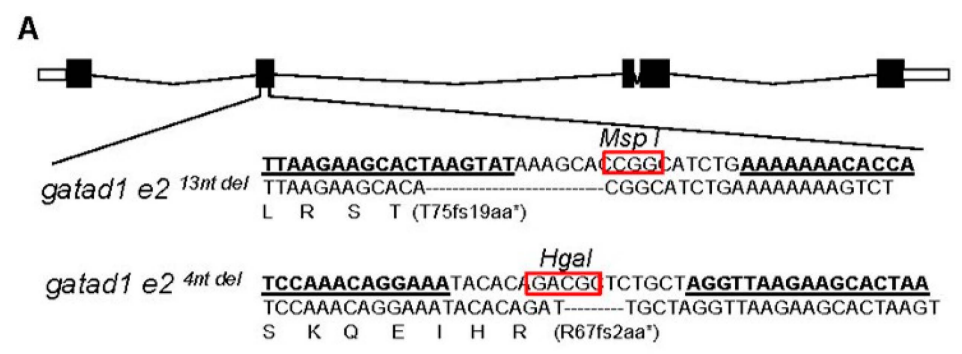

B

C
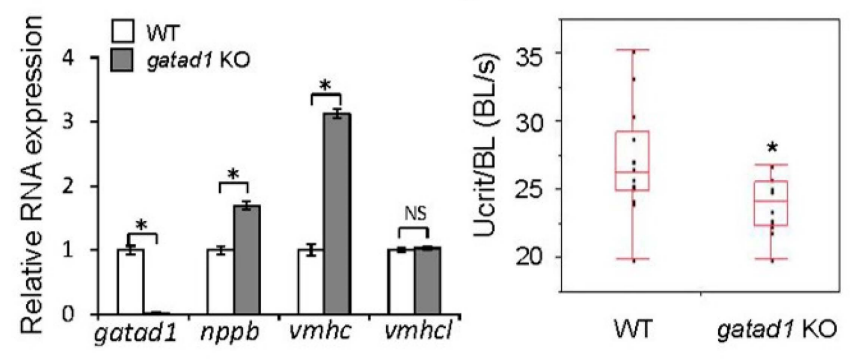

D

E
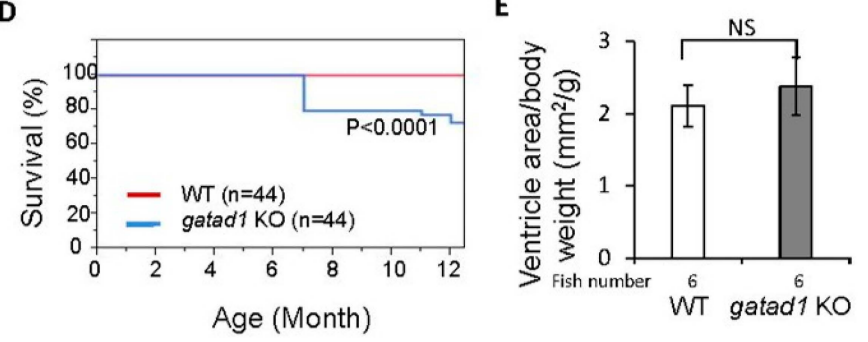

Figure 4. Generating and phenotyping gatad1 knock-out zebrafish. (A) Two TALEN mutants for gatad1 were generated. TALEN RNA binding sites within exon 2 are underlined. The restriction enzyme cut sites that facilitated genotyping are circled. The locations of the 13-bp and 4-bp deletions are shown below the wild type sequence, and the corresponding truncated peptides are indicated; (B) Fetal gene activation in the hearts of gatad1 KO fish. The RNA expression levels of gatad1, nppb, vmhc and vmhcl are shown, as measured by real-time RT-PCR with gapdh as internal control. gatad1 expression was reduced by $98 \%$ in gatad $1^{13 n t d e l}$ homozygous mutant fish. The fetal genes $n p p b$ and vmhc were upregulated in gatad1 mutant fish; (C) Significantly reduced swimming capacity was observed in gatad1 $1^{13 n t d e l}$ homozygous mutant fish at 17 months. gatad $1^{13 n t d e l}$ homozygous mutant fish and age-matched controls were stressed by $0.3 \%$ ethanol from 2 hpf to $48 \mathrm{hpf}$ and then fed with $4 \%$ cholesterol from 2 months of age; (D) Significantly reduced survival rate was observed in gatad1 $1^{13 \text { ntdel }}$ homozygous mutant fish than in age-matched wild type fish after cholesterol and ethanol treatment; (E) Quantification of ventricle area shows no significant difference between wild type and gatad1 ${ }^{13 \text { ntdel }}$ homozygous mutant fish after cholesterol and ethanol treatment. ${ }^{*} p<0.05$; WT, wild type; KO, knock-out. NS, not significant. 


\subsection{Phenotypes in Transgenic Fish Line Expressing GATAD1 Containing the S102P Mutation}

To model the GATAD1-S102P mutation identified in human DCM, we generated two stable transgenic fish lines. While $T g(m y l 7: G A T A D 1)$ expressed human wild type GATAD1 in cardiomyocytes, Tg(myl7:GATAD1S102P) expressed human GATAD1-S102P (Figure 5A). Expression of these ectopic genes was driven specifically in cardiomyocytes by a cardiomyocyte-specific myl7 enhancer [49]. Both transgenic fish lines survived to the adult stage without visually abnormal phenotypes. However, the $\mathrm{Tg}(\mathrm{myl7}$ :GATAD1S102P) fish started to die around 13 months of age, in contrast to Tg(myl7:GATAD1) fish that survived more than 20 months without any deaths (Figure 5B). There was no significant difference in swimming capacity between these two transgenic fish lines at 20 months of age (data not show), but 1 out of $6 \mathrm{Tg}$ (myl7:GATAD1S102P) fish exhibited significantly increased heart size, increased width but decreased density in papillary muscles (Figure 5C). A larger sample size is required to validate this observation.

A

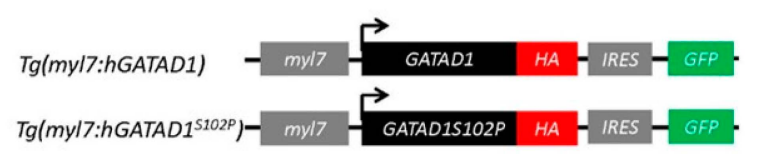

B

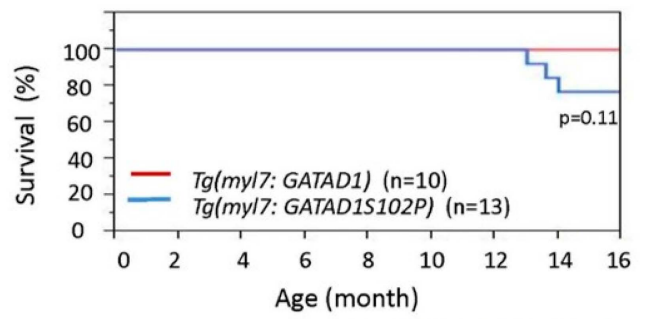

C

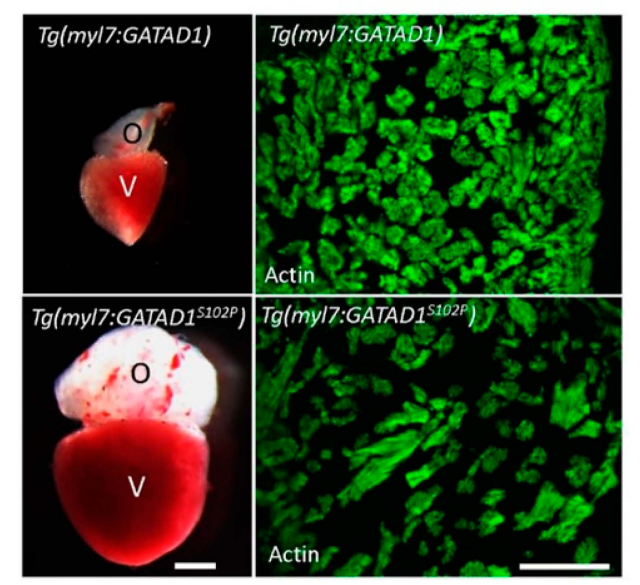

Figure 5. Generating and phenotyping GATAD1 transgenic fish. (A) Schematic illustration of constructs that were used to generate two transgenic fish lines expressing either human wild type GATAD1 or GATAD1-S102P mutation. The GATAD1 gene is flanked by the myl7 enhancer at its $5^{\prime}$ terminal to drive cardiomyocyte-specific expression, and it has an HA tag and IRES-EGFP at its $3^{\prime}$ terminal to facilitate detection of ectopic gene expression and fish propagation; (B) The transgenic fish expressing mutant GATAD1 started to die at approximately 12 months of age, and all transgenic fish expressing wild type GATAD1 were able to survive to 16 months; (C) Severe cardiac hypertrophy in a $\operatorname{Tg}(m y l 7: h G A T A D 1 S 102 P)$ fish. Left panel, images of a heart dissected from a $\operatorname{Tg}(m y l 7: h G A T A D)$ and a $\operatorname{Tg}(m y l 7: h G A T A D 1 S 102 P)$ fish at 17 months of age, separately. The heart from this single $\operatorname{Tg}(m y l 7: h G A T A D 1 S 102 P)$ fish exhibits significantly enlarged ventricle and out flow tract. Right panel, phalloidin staining revealed less dense myofibril and wider myofibril in the heart of this single Tg(myl7:hGATAD1S102P) fish. V, ventricle; O, out flow tract; Scale bar $0.5 \mathrm{~mm}$. 


\section{Discussion}

\subsection{Conservation of Zebrafish as a Model for Cardiomyopathy Genes}

We used GATAD1, a recently identified gene for autosomal recessive DCM [36], as a paradigm to explore adult zebrafish as an in vivo model to validate cardiomyopathy gene mutations with age-dependent penetrance. Our data support the potential of adult zebrafish as an efficient model, but also reveal the limitations of this model that need to be addressed by further technology development.

At the protein level, zebrafish Gatad1 is conserved with human GATAD1. There is $70 \%$ amino acid identity, which is much higher than invertebrate models such as Drosophila. Importantly, over 50 amino acids flanking the human GATAD1 S102P mutation are almost completely identical between zebrafish and human. This high level of conservation justified our efforts to determine if zebrafish modeling would recapitulate the cardiomyopathy phenotype. Interestingly, zebrafish Gatad1 does not contain the serine residue in this position but has an additional two serine residues in positions that correspond to N97 and A106 in human GATAD1. It is possible that the two serine residues in fish species exert similar function as the single serine residue in mammalian GATAD1. Unfortunately, 3-D structure for GATAD1 has not been resolved yet, which prevented us from seeking structural evidence that would support this speculation.

\subsection{Gene Expression Can be Profiled in Zebrafish}

The expression profile of a gene usually provides important insights about its function and possibly its candidacy as a gene for heart disease. The zebrafish model offers the following three strategies to generate expression data for a candidate gene. First, in situ hybridization can be performed to reveal the three-dimensional gene expression profile during zebrafish embryogenesis. This is advantageous because zebrafish embryos develop ex utero and are available in large quantities. In situ data for more than one third of the genome have already been generated and deposited at Zfin (Zfin.org) [50]. Because the gene expression pattern of gatad1 has not been reported in this database, we conducted our own in situ hybridization. We confirmed the cardiac expression of gatad1, and also revealed its expression in head, notochord, gut and pectoral fin. Second, we recently defined the zebrafish cardiac transcriptome at $4 \mathrm{dpf}$ and 6 months using RNAseq technology, which can be used to determine the absolute cardiac expression level of a cardiomyopathy candidate gene [8]. Comparing to the expression of gatad 1 transcript in the embryonic heart, we found that the expression of gatad1 in the adult heart is reduced by half. Third, tissue distribution of a candidate gene can be determined by real time RT-PCR using total RNA extracted from different organs. This is a technology that is also available in other animal models. GATAD1 was also previously named ocular development-associated gene $(O D A G)$, because the gene shows a dynamic expression pattern in the developing eye of mice [35]. Besides the eye, Gatad1 expression was detected in mouse ovary, testis, thymus, lung, kidney, spleen, liver, brain and heart [35]. Our real time RT-PCR data in fish are consistent with this multi-organ expression pattern, suggesting that GATAD1 may have other extra-cardiac functions.

The experimentally accessible zebrafish embryo provides an excellent platform for assessing the subcellular localization of proteins encoded by cardiomyopathy candidate genes. Localization can be studied by injecting a construct encoding a fluorescently tagged protein, making it unnecessary to develop an antibody. Imaging of an embryonic zebrafish heart can be facilitated by a heart isolation technique [42,51]. Of note, ISH can be conducted in sectioned adult zebrafish tissues [52,53], and sublocalization of a protein-of-interest can be followed in the adult stage using a stable transgenic fish line. In this study, we reported that zebrafish Gatad1 localizes to both the nucleus and I-band of the sarcomere. Similar cardiac expression patterns have been reported previously in humans by using a GATAD1 antibody [37]. The subcellular localization suggests future research directions to investigate Gatad1 as both a candidate transcription factor and sarcomeric protein, and uncover the pathogenic mechanism by which it leads to heart failure in DCM. 


\subsection{Genetic Engineering Tools are Available in Zebrafish for Modeling Cardiomyopathy}

One of the most compelling pieces of evidence to validate a cardiomyopathy candidate gene is to recapitulate a cardiomyopathy phenotype in an animal model harboring a similar genetic mutation. At present, generating stable mutants for a candidate gene is no longer a bottleneck in the zebrafish model. Mutants for more than half of genes in the zebrafish genome have been generated by mutagenesis screening and distributed by Zfin. TILLING technology has also been used to produce mutants, and the lines have been distributed by the Zebrafish Mutagenesis Project at Sanger center [54]. Transposon-based mutagenesis screening has been used to produce mutants, and those mutants have been distributed by zFishbook and the International Protein trap consortium [55,56]. If mutants for a particular candidate gene are not available, targeted mutations for any gene can be easily generated by genome editing technology, as exemplified by the present study. If null mutants are needed, large deletions that completely remove the gene locus can be obtained by co-injecting two TALENs or CRISPR/Cas9 RNAs [57,58]. An important limitation of the loss-of-function (LOF) approach is evident in modeling human point mutations. If the missense mutation leads to pathogenesis through a gain-of-function (GOF) mechanism, then the LOF approach will not be able to recapitulate the disease pathogenesis. Knock-in technology would be needed to test these missense mutations, which is feasible in the zebrafish model. It has been shown that homologous recombination can be facilitated by co-injection of TALENs or Cas9 with either short oligos or long double-stranded DNA as a guide template $[33,59,60]$.

As illustrated in the present study of GATAD1, many missense mutations cannot be mimicked by a simple knock-in approach to genetic manipulation. The missing serine residue at position 102 in fish species poses a significant challenge. It is plausible that two strategies can be used to address this challenge. In the first strategy, two steps of knock-in manipulation can be used. A zebrafish line containing a humanized Gatad1 can be initially generated by introducing a serine residue at position 102 and replacing the other two serine residues at positions 97 and 106 with N97 and A106, as in human GATAD1. A S102P mutation can be introduced into position 102 of this humanized fish line. A second strategy, as demonstrated in the present study, uses transgenic technology by directly expressing human GATAD1 with the S102P mutation in the zebrafish heart. Because of the efficient Tol2 transposon system, high transgenic efficiency can be achieved in zebrafish. The spatially-restricted expression of transgenes can be achieved by using tissue-specific enhancers, including the titin enhancer and the myl7 enhancer for myocardium [49,51], the $f l i$ and $k d r l$ enhancers for endocardium [61,62], and the tcf 21 enhancer for epicardium [63]. The temporally-controlled expression of a transgene can be achieved by using the binary cre-loxP system [64]. There are certain limitations associated with transgenic models of cardiomyopathy, however, as have already been recognized in transgenic mouse models of HF in the past decade [65]. The ectopic gene expression level could be higher than a physiologically relevant level lead to non-specific cardiac remodeling process, as shown with GFP [66]. This concern can be partially addressed by including an appropriate control transgenic line, such as a line that expresses wild type human GATAD1.

We found that stable gatad1 homozygous mutants did not exhibit an overt cardiac phenotype during embryogenesis or as adults under baseline conditions. This observation indicated that stable models are critical for assessing late-onset cardiomyopathies. The fact that phenotypes only show up in aged fish models is likely because of the late-onset nature of GATAD1-based cardiomyopathy. It is fully expected that early onset cardiomyopathy can be recapitulated in much younger adult zebrafish. Under imposed stress, we observed HF-like phenotypes in gatad1 KO fish and a tendency to exhibit HF-like phenotypes in GATAD1-S102P transgenic fish that were not evident in the control transgenic line. The former observation suggested a haploinsufficient basis for GATAD1-based cardiomyopathy, which is consistent with the autosomal recessive segregation of DCM in the reported family it was discovered in [36]. On the other hand, the latter observation, if validated by future experimentation with larger sample size, suggests that the S102P mutation might also be able to exert a dominant 
negative effect in the heart. Further investigations in these genetic zebrafish models will clarify the mechanism of GATAD1-based DCM.

\subsection{More Cardiomyopathy Phenotyping Tools are Needed in Adult Zebrafish}

Because zebrafish is a new vertebrate model for cardiomyopathy, methodologies for defining cardiovascular pathophysiology in adult zebrafish are less developed than for rodent models. In this manuscript, we used several phenotyping tools to study the gatad1 models. First, we assessed the survival of the mutant fish. In humans, survival is one of the most important indexes to assess heart failure. The 10-year mortality following diagnosis of dilated cardiomyopathy is approximately 40\% [67]. The low cost of maintaining zebrafish colonies makes it possible to routinely assess survival of a HF model, even for a late-onset disease model such as GATAD1-based DCM. Previously, we demonstrated significantly reduced survival in acquired cardiomyopathy models induced by both anemia and DOX [21,24]. Here, we noted significantly reduced survival in gatad1 $\mathrm{KO}$ fish and a tendency of reduced survival in S102P transgenic fish. Second, we used exercise intolerance as an index for $\mathrm{HF}$ in zebrafish. The treadmill testing is an important index for diagnosis of HF in rodent models [68]. Similarly, we detected reduced swimming capacity in gatad1 KO fish, as would be expected with HF. It is anticipated that exercise intolerance will become an important non-invasive assay for future studies of HF in adult zebrafish. Third, to facilitate the detection of cardiomyopathy phenotypes, we imposed two stresses. Previous studies showed that feeding adult fish a high cholesterol diet induces hypercholesterolemia and atherosclerotic changes [44,45]. Additionally, ethanol exposure interrupts cardiac morphogenesis, causing heart defects in zebrafish embryos [46]. Maternal alcohol consumption can lead to fetal alcohol syndrome including heart defects in both human and animal models [69]. We reasoned that embryonic treatment with lower doses of ethanol would impose a more subtle impairment to the zebrafish heart, which might increase susceptibility to HF in the adult. Indeed, we found that stressing the fish with a high cholesterol diet can distinguish the gatad1 KO from age-matched controls by showing reduced swimming capacity. When fish are stressed by both ethanol treatment and a high cholesterol diet, the gatad1 KO fish exhibit both reduced swimming capacity and increased mortality.

Previously, we have established methodologies to define the hallmarks of cardiomyopathy in adult fish at both the cellular and molecular levels. At the cellular level, it is possible to quantify cardiomyocyte size by immunostaining with $\beta$-catenin or directly quantify isolated cardiomyocytes. Proliferation can be studied by immunostaining with PCNA. Apoptosis can be studied by the TUNNEL assay, and autophagy can be studied by Western blotting with LC3 [19,24]. At the molecular level, it is possible to assess re-activation of fetal genes such as nppa, nppb, and vmhc [8,24]. Here, we showed that both $n p p b$ and $v m h c$ transcripts are upregulated in the gatad1 KO fish hearts. Together with the increased mortality and reduced swimming capacity, our data support GATAD1 as a causative gene for DCM.

In human patients, electrocardiography and echocardiography are two primary diagnostic tests for cardiomyopathy. Despite significant challenges imposed by the small size of the zebrafish heart (1 2 $\mathrm{mm}$ in diameter), both technologies are being developed and becoming feasible. Single lead electrocardiography has been developed to monitor heart conduction in anesthetized adult zebrafish [70,71]. A system that is capable of documenting zebrafish electrocardiograms is now commercially available from iWorx Inc. The technology was further advanced by the use of micro-electrodes [71,72]. Ultrasound technology to assess a zebrafish heart has also been actively developed over the past several years. Pulsed-wave Doppler signals can be readily measured to monitor blood flow in the heart, a surrogate of cardiac output [73,74]. However, measurement of ejection fraction to assess contractile function is relatively difficult to obtain because of limited resolution of the ultrasound machine and the difficulty in determining the border of a small zebrafish heart. 75-MHz-high-frequency ultrasound and Chirp-coded tissue harmonic imaging are being pursued to capture the real-time heart images and to characterize the zebrafish heart $[73,75,76]$. It is 
anticipated that further developments in non-invasive cardiac function technologies will further foster the use of adult zebrafish as an in vivo model for cardiomyopathy.

\section{Conclusions}

Our genetic studies of gatad1 in adult zebrafish highlight the following unique features of this animal for studying cardiomyopathy. First, expression patterns of mRNA and protein at organ, cellular and subcellular levels can be efficiently determined. Secondly, stable gene knock-outs can be efficiently generated to model LOF mutants, and knock-ins and/or transgenes can be generated to study missense mutations that might exert GOF effects. Third, compared to mouse, maintaining a large number of fish colonies is relatively affordable, which is an important consideration when assessing diseases such as cardiomyopathy, which require extended longitudinal studies. Consequently, a single zebrafish lab can feasibly test dozens of candidate genes; such throughput is very difficult, if not impossible, with rodent models. At present, the small size of the zebrafish heart still poses significant challenges for detailed analysis of cardiovascular function. However, this bottleneck is being actively addressed by rapid technological advances. By complementing existing rodent and large mammal models of cardiomyopathy and heart failure, it is anticipated that the emerging adult zebrafish model will create new research opportunities and facilitate gene discovery, mechanistic understanding and therapeutic development for cardiomyopathy.

Supplementary Materials: The following are available online at www.mdpi.com/2308-3425/3/1/6/S1, Figure S1: Tissue-specific expression of gatad1 transcripts was revealed by in situ hybridization in $2 \mathrm{dpf}$ zebrafish embryos.

Acknowledgments: We thank Beninio Gore and Kashia Stragey for managing the zebrafish facility. This work was supported by NIH HL81753 (XX), NIH HL107304 (XX) and the Mayo Foundation (XX).

Author Contributions: T.M.O. and X.X. conceived the idea and designed the experiments; J.Y. and S.S. were responsible for carrying out the experiments and collecting data; J.Y. and X.X. wrote the manuscript; T.M.O. assisted in interpretation of the data and edited the manuscript.

Conflicts of Interest: The authors declare no conflict of interest.

\section{References}

1. Ahmad, F.; Seidman, J.G.; Seidman, C.E. The genetic basis for cardiac remodeling. Annu. Rev. Genom. Hum. Genet. 2005, 6, 185-216. [CrossRef] [PubMed]

2. Maron, B.J.; Maron, M.S.; Semsarian, C. Genetics of hypertrophic cardiomyopathy after 20 years: Clinical perspectives. J. Am. Coll. Cardiol. 2012, 60, 705-715. [CrossRef] [PubMed]

3. Kimura, A. Molecular etiology and pathogenesis of hereditary cardiomyopathy. Circ. J. 2008, 72 (Suppl. A), A38-A48. [CrossRef] [PubMed]

4. Norton, N.; Li, D.; Hershberger, R.E. Next-generation sequencing to identify genetic causes of cardiomyopathies. Curr. Opin. Cardiol. 2012, 27, 214-220. [CrossRef] [PubMed]

5. Lander, E.S.; Linton, L.M.; Birren, B.; Nusbaum, C.; Zody, M.C.; Baldwin, J.; Devon, K.; Dewar, K.; Doyle, M.; FitzHugh, W.; et al. Initial sequencing and analysis of the human genome. Nature 2001, 409, 860-921. [CrossRef] [PubMed]

6. Davis, E.E.; Frangakis, S.; Katsanis, N. Interpreting human genetic variation with in vivo zebrafish assays. Biochim. Biophys. Acta 2014, 1842, 1960-1970. [CrossRef] [PubMed]

7. Staudt, D.; Stainier, D. Uncovering the molecular and cellular mechanisms of heart development using the zebrafish. Annu. Rev. Genet. 2012, 46, 397-418. [CrossRef] [PubMed]

8. Shih, Y.H.; Zhang, Y.; Ding, Y.; Ross, C.A.; Li, H.; Olson, T.M.; Xu, X. The Cardiac Transcriptome and Dilated Cardiomyopathy Genes in Zebrafish. Circ. Cardiovasc. Genet. 2015, 8, 261-269. [CrossRef] [PubMed]

9. Xu, X.; Meiler, S.E.; Zhong, T.P.; Mohideen, M.; Crossley, D.A.; Burggren, W.W.; Fishman, M.C. Cardiomyopathy in zebrafish due to mutation in an alternatively spliced exon of titin. Nat. Genet. 2002, 30, 205-209. [PubMed]

10. Sehnert, A.J.; Huq, A.; Weinstein, B.M.; Walker, C.; Fishman, M.; Stainier, D.Y. Cardiac troponin T is essential in sarcomere assembly and cardiac contractility. Nat. Genet. 2002, 31, 106-110. [CrossRef] [PubMed] 
11. Ruparelia, A.A.; Oorschot, V.; Vaz, R.; Ramm, G.; Bryson-Richardson, R.J. Zebrafish models of BAG3 myofibrillar myopathy suggest a toxic gain of function leading to BAG3 insufficiency. Acta Neuropathol. 2014, 128, 821-833. [CrossRef] [PubMed]

12. Bendig, G.; Grimmler, M.; Huttner, I.G.; Wessels, G.; Dahme, T.; Just, S.; Trano, N.; Katus, H.A.; Fishman, M.C.; Rottbauer, W. Integrin-linked kinase, a novel component of the cardiac mechanical stretch sensor, controls contractility in the zebrafish heart. Genes Dev. 2006, 20, 2361-2372. [CrossRef] [PubMed]

13. Hassel, D.; Dahme, T.; Erdmann, J.; Meder, B.; Huge, A.; Stoll, M.; Just, S.; Hess, A.; Ehlermann, P.; Weichenhan, D.; et al. Nexilin mutations destabilize cardiac Z-disks and lead to dilated cardiomyopathy. Nat. Med. 2009, 15, 1281-1288. [CrossRef] [PubMed]

14. Kessler, M.; Rottbauer, W.; Just, S. Recent progress in the use of zebrafish for novel cardiac drug discovery. Expert Opin. Drug Discov. 2015, 10, 1231-1241. [CrossRef] [PubMed]

15. Becker, J.R.; Robinson, T.Y.; Sachidanandan, C.; Kelly, A.E.; Coy, S.; Peterson, R.T.; MacRae, C.A. In vivo natriuretic peptide reporter assay identifies chemical modifiers of hypertrophic cardiomyopathy signalling. Cardiovasc. Res. 2012, 93, 463-470. [CrossRef] [PubMed]

16. Amsterdam, A.; Nissen, R.M.; Sun, Z.; Swindell, E.C.; Farrington, S.; Hopkins, N. Identification of 315 genes essential for early zebrafish development. Proc. Natl. Acad. Sci. USA 2004, 101, 12792-12797. [CrossRef] [PubMed]

17. Kok, F.O.; Shin, M.; Ni, C.W.; Gupta, A.; Grosse, A.S.; van Impel, A.; Kirchmaier, B.C.; Peterson-Maduro, J.; Kourkoulis, G.; Male, I.; et al. Reverse genetic screening reveals poor correlation between morpholino-induced and mutant phenotypes in zebrafish. Dev. Cell 2015, 32, 97-108. [CrossRef] [PubMed]

18. Rossi, A.; Kontarakis, Z.; Gerri, C.; Nolte, H.; Holper, S.; Kruger, M.; Stainier, D.Y. Genetic compensation induced by deleterious mutations but not gene knockdowns. Nature 2015, 524, 230-233. [CrossRef] [PubMed]

19. Sun, X.; Hoage, T.; Bai, P.; Ding, Y.; Chen, Z.; Zhang, R.; Huang, W.; Jahangir, A.; Paw, B.; Li, Y.G.; et al. Cardiac hypertrophy involves both myocyte hypertrophy and hyperplasia in anemic zebrafish. PLoS ONE 2009, 4, e6596. [CrossRef] [PubMed]

20. Paw, B.H.; Davidson, A.J.; Zhou, Y.; Li, R.; Pratt, S.J.; Lee, C.; Trede, N.S.; Brownlie, A.; Donovan, A.; Liao, E.C.; et al. Cell-specific mitotic defect and dyserythropoiesis associated with erythroid band 3 deficiency. Nat. Genet. 2003, 34, 59-64. [CrossRef] [PubMed]

21. Sun, X.; Xu, X. Anemic Zebrafish Models of Cardiomyopathy. In Methods Pharmacol. Toxicol.; 2012; pp. 41-54.

22. Hayward, R.; Hydock, D.S. Doxorubicin cardiotoxicity in the rat: An in vivo characterization. J. Am. Assoc. Lab. Anim. Sci. 2007, 46, 20-32. [PubMed]

23. Christiansen, S.; Autschbach, R. Doxorubicin in experimental and clinical heart failure. Eur. J. Cardiothorac. Surg. 2006, 30, 611-616. [CrossRef] [PubMed]

24. Ding, Y.; Sun, X.; Huang, W.; Hoage, T.; Redfield, M.; Kushwaha, S.; Sivasubbu, S.; Lin, X.; Ekker, S.; $\mathrm{Xu}, \mathrm{X}$. Haploinsufficiency of target of rapamycin attenuates cardiomyopathies in adult zebrafish. Circ. Res. 2011, 109, 658-669. [CrossRef] [PubMed]

25. Asimaki, A.; Kapoor, S.; Plovie, E.; Karin Arndt, A.; Adams, E.; Liu, Z.; James, C.A.; Judge, D.P.; Calkins, H.; Churko, J.; et al. Identification of a new modulator of the intercalated disc in a zebrafish model of arrhythmogenic cardiomyopathy. Sci. Transl. Med. 2014, 6, 240ra274. [CrossRef] [PubMed]

26. Haffter, P.; Granato, M.; Brand, M.; Mullins, M.C.; Hammerschmidt, M.; Kane, D.A.; Odenthal, J.; van Eeden, F.J.; Jiang, Y.J.; Heisenberg, C.P.; et al. The identification of genes with unique and essential functions in the development of the zebrafish, Danio rerio. Development 1996, 123, 1-36. [PubMed]

27. Driever, W.; Solnica-Krezel, L.; Schier, A.F.; Neuhauss, S.C.; Malicki, J.; Stemple, D.L.; Stainier, D.Y.; Zwartkruis, F.; Abdelilah, S.; Rangini, Z.; et al. A genetic screen for mutations affecting embryogenesis in zebrafish. Development 1996, 123, 37-46. [PubMed]

28. Wang, D.; Jao, L.E.; Zheng, N.; Dolan, K.; Ivey, J.; Zonies, S.; Wu, X.; Wu, K.; Yang, H.; Meng, Q.; et al. Efficient genome-wide mutagenesis of zebrafish genes by retroviral insertions. Proc. Natl. Acad. Sci. USA 2007, 104, 12428-12433. [CrossRef] [PubMed]

29. Campbell, J.M.; Hartjes, K.A.; Nelson, T.J.; Xu, X.; Ekker, S.C. New and TALENted Genome Engineering Toolbox. Circ. Res. 2013, 113, 571-587. [CrossRef] [PubMed]

30. Cade, L.; Reyon, D.; Hwang, W.Y.; Tsai, S.Q.; Patel, S.; Khayter, C.; Joung, J.K.; Sander, J.D.; Peterson, R.T.; Yeh, J.R. Highly efficient generation of heritable zebrafish gene mutations using homo- and heterodimeric TALENs. Nucleic Acids Res. 2012, 40, 8001-8010. [CrossRef] [PubMed] 
31. Dahlem, T.J.; Hoshijima, K.; Jurynec, M.J.; Gunther, D.; Starker, C.G.; Locke, A.S.; Weis, A.M.; Voytas, D.F.; Grunwald, D.J. Simple methods for generating and detecting locus-specific mutations induced with TALENs in the zebrafish genome. PLoS Genet. 2012, 8, e1002861. [CrossRef] [PubMed]

32. Auer, T.O.; Duroure, K.; de Cian, A.; Concordet, J.P.; del Bene, F. Highly efficient CRISPR/Cas9-mediated knock-in in zebrafish by homology-independent DNA repair. Genome Res. 2014, 24, 142-153. [CrossRef] [PubMed]

33. Shin, J.; Chen, J.; Solnica-Krezel, L. Efficient homologous recombination-mediated genome engineering in zebrafish using TALE nucleases. Development 2014, 141, 3807-3818. [CrossRef] [PubMed]

34. Kwan, K.M.; Fujimoto, E.; Grabher, C.; Mangum, B.D.; Hardy, M.E.; Campbell, D.S.; Parant, J.M.; Yost, H.J.; Kanki, J.P.; Chien, C.B. The Tol2kit: A multisite gateway-based construction kit for Tol2 transposon transgenesis constructs. Dev. Dyn. 2007, 236, 3088-3099. [CrossRef] [PubMed]

35. Tsuruga, T.; Kanamoto, T.; Kato, T.; Yamashita, H.; Miyagawa, K.; Mishima, H.K. Ocular development-associated gene (ODAG), a novel gene highly expressed in ocular development. Gene 2002, 290, 125-130. [CrossRef]

36. Sasaki, T.; Watanabe, W.; Muranishi, Y.; Kanamoto, T.; Aihara, M.; Miyazaki, K.; Tamura, H.; Saeki, T.; Oda, H.; Souchelnytskyi, N.; et al. Elevated intraocular pressure, optic nerve atrophy, and impaired retinal development in ODAG transgenic mice. Investig. Ophthalmol. Vis. Sci. 2009, 50, 242-248. [CrossRef] [PubMed]

37. Theis, J.L.; Sharpe, K.M.; Matsumoto, M.E.; Chai, H.S.; Nair, A.A.; Theis, J.D.; de Andrade, M.; Wieben, E.D.; Michels, V.V.; Olson, T.M. Homozygosity mapping and exome sequencing reveal GATAD1 mutation in autosomal recessive dilated cardiomyopathy. Circ. Cardiovasc. Genet. 2011, 4, 585-594. [CrossRef] [PubMed]

38. Heidecker, B.; Lamirault, G.; Kasper, E.K.; Wittstein, I.S.; Champion, H.C.; Breton, E.; Russell, S.D.; Hall, J.; Kittleson, M.M.; Baughman, K.L.; et al. The gene expression profile of patients with new-onset heart failure reveals important gender-specific differences. Eur. Heart J. 2010, 31, 1188-1196. [CrossRef] [PubMed]

39. Cermak, T.; Doyle, E.L.; Christian, M.; Wang, L.; Zhang, Y.; Schmidt, C.; Baller, J.A.; Somia, N.V.; Bogdanove, A.J.; Voytas, D.F. Efficient design and assembly of custom TALEN and other TAL effector-based constructs for DNA targeting. Nucleic Acids Res. 2011, 39, e82. [CrossRef] [PubMed]

40. Ma, A.C.; Lee, H.B.; Clark, K.J.; Ekker, S.C. High efficiency In vivo genome engineering with a simplified 15-RVD GoldyTALEN design. PLoS ONE 2013, 8, e65259. [CrossRef] [PubMed]

41. Sun, X.; Zhang, R.; Lin, X.; Xu, X. Wnt3a regulates the development of cardiac neural crest cells by modulating expression of cysteine-rich intestinal protein 2 in rhombomere 6. Circ. Res. 2008, 102, 831-839. [CrossRef] [PubMed]

42. Yang, J.; Xu, X. Immunostaining of dissected zebrafish embryonic heart. J. Vis. Exp. JoVE 2012, e3510. [CrossRef] [PubMed]

43. Sander, J.D.; Zaback, P.; Joung, J.K.; Voytas, D.F.; Dobbs, D. Zinc Finger Targeter (ZiFiT): An engineered zinc finger/target site design tool. Nucleic Acids Res. 2007, 35, W599-W605. [CrossRef] [PubMed]

44. Fang, L.; Green, S.R.; Baek, J.S.; Lee, S.H.; Ellett, F.; Deer, E.; Lieschke, G.J.; Witztum, J.L.; Tsimikas, S.; Miller, Y.I. In vivo visualization and attenuation of oxidized lipid accumulation in hypercholesterolemic zebrafish. J. Clin. Investig. 2011, 121, 4861-4869. [CrossRef] [PubMed]

45. Yoon, Y.; Yoon, J.; Jang, M.Y.; Na, Y.; Ko, Y.; Choi, J.H.; Seok, S.H. High cholesterol diet induces IL-1beta expression in adult but not larval zebrafish. PLOS ONE 2013, 8, e66970.

46. Sarmah, S.; Marrs, J.A. Complex cardiac defects after ethanol exposure during discrete cardiogenic events in zebrafish: Prevention with folic acid. Dev. Dyn. 2013, 242, 1184-1201. [CrossRef] [PubMed]

47. Sun, Y.; Fang, Y.; Xu, X.; Lu, G.; Chen, Z. Evidence of an Association between Age-Related Functional Modifications and Pathophysiological Changes in Zebrafish Heart. Gerontology 2015, 61, 435-447. [CrossRef] [PubMed]

48. Wang, J.; Panakova, D.; Kikuchi, K.; Holdway, J.E.; Gemberling, M.; Burris, J.S.; Singh, S.P.; Dickson, A.L.; Lin, Y.F.; Sabeh, M.K.; et al. The regenerative capacity of zebrafish reverses cardiac failure caused by genetic cardiomyocyte depletion. Development 2011, 138, 3421-3430. [CrossRef] [PubMed]

49. Huang, C.J.; Tu, C.T.; Hsiao, C.D.; Hsieh, F.J.; Tsai, H.J. Germ-line transmission of a myocardium-specific GFP transgene reveals critical regulatory elements in the cardiac myosin light chain 2 promoter of zebrafish. Dev. Dyn. 2003, 228, 30-40. [CrossRef] [PubMed] 
50. Thisse, B.; Thisse, C. In situ hybridization on whole-mount zebrafish embryos and young larvae. Methods Mol. Biol. 2014, 1211, 53-67. [PubMed]

51. Yang, J.; Xu, X. Alpha-Actinin2 is required for the lateral alignment of $\mathrm{Z}$ discs and ventricular chamber enlargement during zebrafish cardiogenesis. FASEB J. 2012, 26, 4230-4242. [CrossRef] [PubMed]

52. Schnabel, K.; Wu, C.C.; Kurth, T.; Weidinger, G. Regeneration of cryoinjury induced necrotic heart lesions in zebrafish is associated with epicardial activation and cardiomyocyte proliferation. PLOS ONE 2011, 6, e18503. [CrossRef] [PubMed]

53. Chablais, F.; Jazwinska, A. The regenerative capacity of the zebrafish heart is dependent on TGFbeta signaling. Development 2012, 139, 1921-1930. [CrossRef] [PubMed]

54. Kettleborough, R.N.; Busch-Nentwich, E.M.; Harvey, S.A.; Dooley, C.M.; de Bruijn, E.; van Eeden, F.; Sealy, I.; White, R.J.; Herd, C.; Nijman, I.J.; et al. A systematic genome-wide analysis of zebrafish protein-coding gene function. Nature 2013, 496, 494-497. [CrossRef] [PubMed]

55. Clark, K.J.; Balciunas, D.; Pogoda, H.M.; Ding, Y.; Westcot, S.E.; Bedell, V.M.; Greenwood, T.M.; Urban, M.D.; Skuster, K.J.; Petzold, A.M.; et al. In vivo protein trapping produces a functional expression codex of the vertebrate proteome. Nat. Methods 2011, 8, 506-515. [CrossRef] [PubMed]

56. Clark, K.J.; Argue, D.P.; Petzold, A.M.; Ekker, S.C. Zfishbook: Connecting you to a world of zebrafish revertible mutants. Nucleic Acids Res. 2012, 40, D907-D911. [CrossRef] [PubMed]

57. Xiao, A.; Wang, Z.; Hu, Y.; Wu, Y.; Luo, Z.; Yang, Z.; Zu, Y.; Li, W.; Huang, P.; Tong, X.; et al. Chromosomal deletions and inversions mediated by TALENs and CRISPR/Cas in zebrafish. Nucleic Acids Res. 2013, 41, e141. [CrossRef] [PubMed]

58. Gupta, A.; Hall, V.L.; Kok, F.O.; Shin, M.; McNulty, J.C.; Lawson, N.D.; Wolfe, S.A. Targeted chromosomal deletions and inversions in zebrafish. Genome Res. 2013, 23, 1008-1017. [CrossRef] [PubMed]

59. Bedell, V.M.; Wang, Y.; Campbell, J.M.; Poshusta, T.L.; Starker, C.G.; Krug, R.G., 2nd; Tan, W.; Penheiter, S.G.; Ma, A.C.; Leung, A.Y.; et al. In vivo genome editing using a high-efficiency TALEN system. Nature 2012, 491, 114-118. [CrossRef] [PubMed]

60. Zu, Y.; Tong, X.; Wang, Z.; Liu, D.; Pan, R.; Li, Z.; Hu, Y.; Luo, Z.; Huang, P.; Wu, Q.; et al. TALEN-mediated precise genome modification by homologous recombination in zebrafish. Nat. Methods 2013, 10, 329-331. [CrossRef] [PubMed]

61. Lawson, N.D.; Weinstein, B.M. In vivo imaging of embryonic vascular development using transgenic zebrafish. Dev. Biol. 2002, 248, 307-318. [CrossRef] [PubMed]

62. Choi, J.; Dong, L.; Ahn, J.; Dao, D.; Hammerschmidt, M.; Chen, J.N. FoxH1 negatively modulates flk1 gene expression and vascular formation in zebrafish. Dev. Biol. 2007, 304, 735-744. [CrossRef] [PubMed]

63. Kikuchi, K.; Gupta, V.; Wang, J.; Holdway, J.E.; Wills, A.A.; Fang, Y.; Poss, K.D. tcf21+ epicardial cells adopt non-myocardial fates during zebrafish heart development and regeneration. Development 2011, 138, 2895-2902. [CrossRef] [PubMed]

64. Hans, S.; Kaslin, J.; Freudenreich, D.; Brand, M. Temporally-controlled site-specific recombination in zebrafish. PLoS ONE 2009, 4, e4640. [CrossRef] [PubMed]

65. Davis, J.; Maillet, M.; Miano, J.M.; Molkentin, J.D. Lost in transgenesis: A user's guide for genetically manipulating the mouse in cardiac research. Circ. Res. 2012, 111, 761-777. [CrossRef] [PubMed]

66. Molkentin, J.D.; Robbins, J. With great power comes great responsibility: Using mouse genetics to study cardiac hypertrophy and failure. J. Mol. Cell. Cardiol. 2009, 46, 130-136. [CrossRef] [PubMed]

67. Simpson, S.; Edwards, J.; Ferguson-Mignan, T.F.; Cobb, M.; Mongan, N.P.; Rutland, C.S. Genetics of Human and Canine Dilated Cardiomyopathy. Int. J. Genom. 2015, 2015, 204823. [CrossRef] [PubMed]

68. James, J.F.; Hewett, T.E.; Robbins, J. Cardiac physiology in transgenic mice. Circ. Res. 1998, 82, $407-415$. [CrossRef] [PubMed]

69. Burd, L.; Deal, E.; Rios, R.; Adickes, E.; Wynne, J.; Klug, M.G. Congenital heart defects and fetal alcohol spectrum disorders. Congenit. Heart Disease 2007, 2, 250-255. [CrossRef] [PubMed]

70. Milan, D.J.; Jones, I.L.; Ellinor, P.T.; MacRae, C.A. In vivo recording of adult zebrafish electrocardiogram and assessment of drug-induced QT prolongation. Am. J. Physiol. Heart Circ. Physiol. 2006, 291, H269-H273. [CrossRef] [PubMed]

71. Sun, P.; Zhang, Y.; Yu, F.; Parks, E.; Lyman, A.; Wu, Q.; Ai, L.; Hu, C.H.; Zhou, Q.; Shung, K.; et al. Micro-electrocardiograms to study post-ventricular amputation of zebrafish heart. Ann. Biomed. Eng. 2009, 37, 890-901. [CrossRef] [PubMed] 
72. Yu, F.; Zhao, Y.; Gu, J.; Quigley, K.L.; Chi, N.C.; Tai, Y.C.; Hsiai, T.K. Flexible microelectrode arrays to interface epicardial electrical signals with intracardial calcium transients in zebrafish hearts. Biomed. Microdevices 2012, 14, 357-366. [CrossRef] [PubMed]

73. Sun, L.; Lien, C.L.; Xu, X.; Shung, K.K. In vivo cardiac imaging of adult zebrafish using high frequency ultrasound (45-75 MHz). Ultrasound Med. Biol. 2008, 34, 31-39. [CrossRef] [PubMed]

74. Lee, J.; Cao, H.; Kang, B.J.; Jen, N.; Yu, F.; Lee, C.A.; Fei, P.; Park, J.; Bohlool, S.; Lash-Rosenberg, L.; et al. Hemodynamics and ventricular function in a zebrafish model of injury and repair. Zebrafish 2014, 11, 447-454. [CrossRef] [PubMed]

75. Liu, T.Y.; Lee, P.Y.; Huang, C.C.; Sun, L.; Shung, K.K. A study of the adult zebrafish ventricular function by retrospective Doppler-gated ultrahigh-frame-rate echocardiography. IEEE Trans. Ultrason. Ferroelectr. Freq. Control 2013, 60, 1827-1837. [CrossRef] [PubMed]

76. Bosisio, M.R.; Hasquenoph, J.M.; Sandrin, L.; Laugier, P.; Bridal, S.L.; Yon, S. Real-time chirp-coded imaging with a programmable ultrasound biomicroscope. IEEE Trans. Biomed. Eng. 2010, 57, 654-664. [CrossRef] [PubMed]

(C) 2016 by the authors; licensee MDPI, Basel, Switzerland. This article is an open access article distributed under the terms and conditions of the Creative Commons by Attribution (CC-BY) license (http:/ / creativecommons.org/licenses/by/4.0/). 